\title{
The influence of impedance mismatch of the medium with gradient change of impedance on acoustic characteristics
}

\author{
Bo $\mathrm{Hu}^{1,2, *}$, Moran $\mathrm{Wang}^{2}$, and Yimei $\mathrm{Du}^{2}$ \\ ${ }^{1}$ Acoustic Science and Technology Laboratory, Harbin Engineering University, Harbin 150001, China \\ ${ }^{2}$ College of Underwater Acoustic Engineering, Harbin Engineering University, Harbin 150001, China
}

\begin{abstract}
Medium with gradient change of acoustic impedance is a new type acoustic structure. It is composed of several materials and has a continuous gradient composition and structure whose specific acoustic impedance varies smoothly across the layer. It has been used in various practical situations such as acoustic rectifiers, medical devices, weakening vibration and reducing noise. The purpose of this paper is to investigate the effect of boundary impedance mismatch on acoustic characteristics of gradient impedance media. The propagation mechanism of acoustic waves in medium with gradient change of specific acoustic impedance was calculated by transfer matrix method. By setting the different ratios of the acoustic impedance at interfaces of matching layer, the relationship between specific acoustic impedance mismatch and acoustic characteristics are derived and the corresponding conclusions are drawn. These conclusions provide an idea for the preparation of relevant medium.
\end{abstract}

\section{Introduction}

Recently, acoustic materials have attracted great research attention due to their wide applicability, such as medical treatment, construction industry and military field[1]. Among all these fields, medium with gradient change of acoustic impedance has been developing rapidly because of its unusual material parameters[2]. Acoustic characteristics of different acoustic impedance distribution media was calculated by Pedersen[3]. The sound reflection and transmission of the anisotropic medium with multilayer composite structure was calculated by Caviglia[4]. Li suggested a genetic algorithm approach to optimizing the effective material parameters of an acoustic metamaterial to minimize acoustic impedance mismatch[5]. Feng presented the micro fabrication of an acoustic impedance gradient matching layer on a spherically-shaped piezoelectric ultrasonic transducer[6]. Kimura proposed a wideband polymer ultrasonic transducer having acoustic impedance matching layers[7]. The performance of acoustic absorbers can be improved by providing a smooth transition from the impedance of air to the impedance of the absorbing material in question was proposed by Ouahabi[8]. Liu realized full-angle negative reflection by a gradient acoustic metasurface to to reduce the harm of noise to human body[9]. Xu used epoxy resin as matrix and aluminum and iron powders as filling components to make the acoustic gradient composites, and investigated the density and acoustic properties of the composites in the gradient direction[10].

Despite numerous advantages, medium with gradient change of acoustic impedance is difficult to match the acoustic impedance on both sides of the medium due to its complex physical parameter distribution. Acoustic impedance mismatch is a common problem in the field of acoustics, which leads to the reflection of acoustic energy at each interface when the incident wave propagates in a complex system. In this paper, the propagation law of acoustic waves in the medium with gradient change of acoustic impedance is derived. By comparing the numerical result of different acoustic impedance mismatch cases, the influence of acoustic impedance mismatch on the acoustic characteristics is obtained. The conclusions are valid and have great potential in various applications.

\section{Theoretical analysis}

There are two semi-infinite homogeneous medium labled medium I and medium III, respectively. Medium II is a medium with gradient change of acoustic impedance located between medium I and medium III. The incident plane wave is propagating in direction from medium I into the medium II and then transmits into medium III.

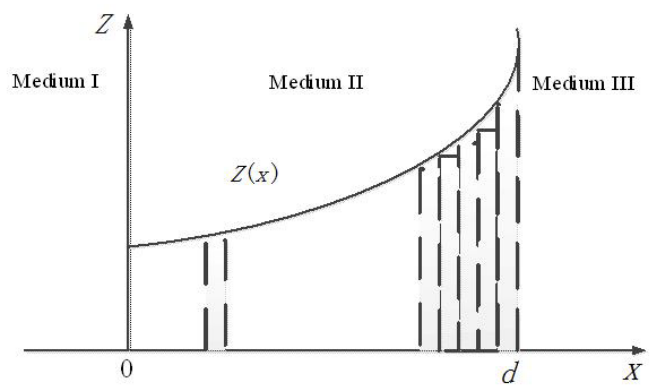

Fig. 1. Impedance distribution model of the medium with gradient change of impedance.

Based on the definition of acoustic impedance, transfer matrix and Euler equation, this paper obtains the

Corresponding author: kidd1105@sina.com 
relationship between acoustic impedance change rate and sound pressure.

The motion equation of small amplitude sound waves in ideal fluid medium takes the form

$$
\rho \frac{\partial v(x, t)}{\partial t}=-\frac{\partial p(x, t)}{\partial x}
$$

where $p$ is the particle pressure in medium, $v$ is the particle velocity and $\rho$ is density. According to the definition of the adiabatic volume elasticity coefficient $K_{s}$, it can be obtained the relationship of the sound pressure $p$ and the relative increment of the volume $d V / V$

$$
p(x, t)=-K_{s} \frac{d V}{V}=-K_{s} \Delta
$$

where $\Delta$ is the relative volume fluctuation by the sound disturbance, for the condition of the plane wave

$$
\Delta=\frac{d V}{V}=-\frac{d \rho}{\rho}=\frac{\partial \xi}{\partial x}
$$

where $\xi$ is displacement, and then substitute the above equation into (2)

$$
p(x, t)=-K_{s} \frac{\partial \xi(x, t)}{\partial x}
$$

In the ideal fluid medium, the solution of the one-dimensional wave equation has the following form

$$
\begin{aligned}
p(x, t) & =A e^{j \omega(t-x / \tilde{c})}+B e^{j \omega(t+x / \tilde{c})} \\
& =p^{+}(x, t)+p^{-}(x, t)
\end{aligned}
$$

where $\tilde{\boldsymbol{c}}$ is the complex sound speed. $p^{+}(x, t)$ represents the wave in $x$ positive direction and $p^{-}(x, t)$ represents the wave in $x$ negative direction. Define the wave impedance $Z(x)$ at some point in the sound field

$$
Z(x)=\rho \tilde{c}=\frac{K}{\tilde{c}}=\frac{p(x, t)}{v(x, t)}
$$

where $K$ is the complex bulk modulus, $\tilde{c}=(K / \rho)^{1 / 2}$. Applying the Euler formula to Eq.4, the particle velocity of the following form is obtained

$$
v(x, t)=\frac{1}{\rho \tilde{c}}\left(A e^{j \omega(t-x / \tilde{c})}-B e^{j \omega(t+x / \tilde{c})}\right)
$$

That is

$$
Z(x) v(x, t)=p^{+}(x, t)-p^{-}(x, t)
$$

From Eq.4 and Eq.6, we find

$$
\begin{aligned}
& p^{+}(x, t)=(p(x, t)+Z(x) v(x, t)) / 2 \\
& p^{-}(x, t)=(p(x, t)-Z(x) v(x, t)) / 2 \\
& \frac{\partial}{\partial x} p^{+}(x, t)=\frac{1}{2 Z(x, t)} \frac{\partial Z(x, t)}{\partial x}\left(p^{+}(x, t)-p^{-}(x, t)\right) \\
&-j \frac{\omega}{\tilde{c}} p^{+}(x, t) \\
& \frac{\partial}{\partial x} p^{-}(x, t)=\frac{1}{2 Z(x, t)} \frac{\partial Z(x, t)}{\partial x}\left(p^{+}(x, t)+p^{-}(x, t)\right) \\
&+j \frac{\omega}{\tilde{c}} p^{-}(x, t)
\end{aligned}
$$

Representing Eq.4 as a vector form

$$
p(x, t)=\left[\begin{array}{ll}
1 & 0 \\
0 & 1
\end{array}\right]\left[\begin{array}{l}
p^{+}(x, t) \\
p^{-}(x, t)
\end{array}\right]
$$

Differentiating the above equation and inserting the result into Eq.9 and Eq.10, we will find

$$
\begin{aligned}
\frac{\partial p(x, t)}{\partial x} & =\left[j \frac{\omega}{\tilde{c}}\left[\begin{array}{cc}
-1 & 0 \\
0 & 1
\end{array}\right]+\frac{1}{2 Z(x)} \frac{\partial Z(x)}{\partial x}\left[\begin{array}{cc}
1 & -1 \\
-1 & 1
\end{array}\right]\right]\left[\begin{array}{c}
p^{+}(x, t) \\
p^{-}(x, t)
\end{array}\right] \\
& =\left[j \frac{\omega}{\tilde{c}}\left[\begin{array}{cc}
-1 & 0 \\
0 & 1
\end{array}\right]+a(x)\left[\begin{array}{cc}
1 & -1 \\
-1 & 1
\end{array}\right]\right] p(x, t) \\
& =C(x) p(x, t)
\end{aligned}
$$

where $\alpha(x)$ is the relative variation of the medium acoustic impedance

$$
\begin{gathered}
a(x)=\frac{1}{2 Z(x)} \frac{\partial Z(x)}{\partial x} \\
C(x)=j \frac{\omega}{\tilde{c}}\left[\begin{array}{cc}
-1 & 0 \\
0 & 1
\end{array}\right]+a(x)\left[\begin{array}{cc}
1 & -1 \\
-1 & 1
\end{array}\right]
\end{gathered}
$$

Using transfer matrix to establish transfer function of medium II.

Matrix $G$ is expressed as

$$
\begin{gathered}
G=e^{-\beta \Delta t}\left[\begin{array}{cc}
E & \frac{\beta}{\omega^{*}} \sin \left(\omega^{*} \Delta t\right) \\
\frac{\beta}{\omega^{*}} \sin \left(\omega^{*} \Delta t\right) & F \\
\beta=\frac{1}{2 Z} \frac{\partial Z}{\partial t}
\end{array}\right] \\
E=\cos \left(\omega^{*} \Delta t\right)+j \frac{\omega}{\omega^{*}} \sin \left(\omega^{*} \Delta t\right) \\
F=\cos \left(\omega^{*} \Delta t\right)-j \frac{\omega}{\omega^{*}} \sin \left(\omega^{*} \Delta t\right)
\end{gathered}
$$

where $\omega^{*}=\sqrt{\omega^{2}+\beta^{2}}$. The transfer matrix $G_{t}$ for the whole medium II takes the following form

$$
G_{t}=\prod_{i=1}^{n} G_{i}
$$

Considering the boundary conditions at the left and right boundary of medium II

$$
M=Q_{1} G_{t} Q_{2}
$$

where $Q_{1}$ and $Q_{1}$ are the boundary transfer matrix of medium II.

Here define

$$
M=\left[\begin{array}{ll}
m_{11} & m_{12} \\
m_{21} & m_{22}
\end{array}\right]
$$

Then the pressure reflection coefficient $R$ is represented as

$$
R=\frac{m_{21}}{m_{11}}
$$

\section{Simulation results}

This part simulates the acoustic characteristics of the three mismatch cases according to the above theoretical formula, compares the results of different situations and 
draws conclusions. In this part, medium I is water, medium II is medium with gradient change of acoustic impedance, medium III is aluminum.

\subsection{Acoustic impedance mismatch between boundary of medium and water}

In this section, the acoustic characteristics of acoustic impedance mismatch between boundary of medium and water are attained by comparing the numerical results of different mismatch cases.

$\mathrm{Z}_{0}$ represents the acoustic impedance at the interface between the medium with gradient change of acoustic impedance and water, $Z_{\mathrm{w}}$ means the acoustic impedance of water. Four mismatch cases are as follows: case 1: $Z_{0} / Z_{w}=0.6$, case $2: Z_{0} / Z_{w}=0.8$, case $3: Z_{0} / Z_{w}=1.2$, case 4 : $Z_{0} / Z_{\mathrm{w}}=1.4$, and a matching case: $Z_{0} / Z_{\mathrm{w}}=1.0$. The acoustic impedance at the boundary of the medium and aluminum is the same as the acoustic impedance of aluminum. Acoustic impedance distributions of the medium are shown in Fig.2(a). The simulation results for the acoustic characteristics of five cases within the frequency range of $0-100 \mathrm{kHz}$ are shown in Fig.2(b).

In Fig.2(b), five cases are almost unchanged within the frequency range of $0-5 \mathrm{kHz}$. In the frequency range of $5 \mathrm{kHz}-10 \mathrm{kHz}$, the reflection coefficients of case 3 and case 4 are always less than other three cases, at the frequency of $10 \mathrm{kHz}$, the reflection coefficients are around 0.6. The reflection coefficients of case 1 and case 2 are always more than other three cases, at the frequency of $10 \mathrm{kHz}$ the reflection coefficients are around 0.8 . In the frequency range of $10 \mathrm{kHz}-100 \mathrm{kHz}$ all five cases have varying degrees of oscillations. To compare the acoustic characteristics of various acoustic impedance mismatch cases, the average values of the reflection coefficient of each frequency are calculated.

$\bar{R}_{n}(\mathrm{n}=1,2,3, \ldots)$ mean the average values of the reflec tion coefficients. After calculation, $\bar{R}_{1}=0.369, \bar{R}_{2}$ $=0.2665, \bar{R}_{3}=0.2246, \bar{R}_{4}=0.2599$. The average values of the reflection coefficients obtained from the matching case is 0.2206 . According to observations, $\bar{R}_{1}$ is larger than other cases, while $\bar{R}_{2}, \bar{R}_{3}$ and $\bar{R}_{4}$ are close to the data of matching case. It makes no sense to research case 2 due to it will increase the difficulty of preparing medium. We explore the relationship between the degree of acoustic impedance mismatch and the acoustic characteristics of the medium. The aim is to search the appropriate mismatch range that reduces the difficulty of preparing medium with gradient change of acoustic impedance. In order to calculate the effects of different acoustic impedance mismatch on the acoustic characteristics of the medium, we define $a=\left(Z_{0}-Z_{w}\right) / Z_{w}$, which means the degree of acoustic impedance mismatch between the medium and water. The reflection coefficients are simulated within the frequency range of $0-100 \mathrm{kHz}$, the results are shown in Fig.3.

Within the frequency range of $0-10 \mathrm{kHz}$, five cases are almost unchanged. As frequency increases, all the cases have different degrees of oscillation. For comparison, a calculation criterion for the degree of mismatch $\Delta$ is defined. The values corresponding to each mismatch cases are shown in Table 1.

$$
\Delta=\left|\frac{R-R_{m}}{R_{m}}\right| \times 100 \%
$$

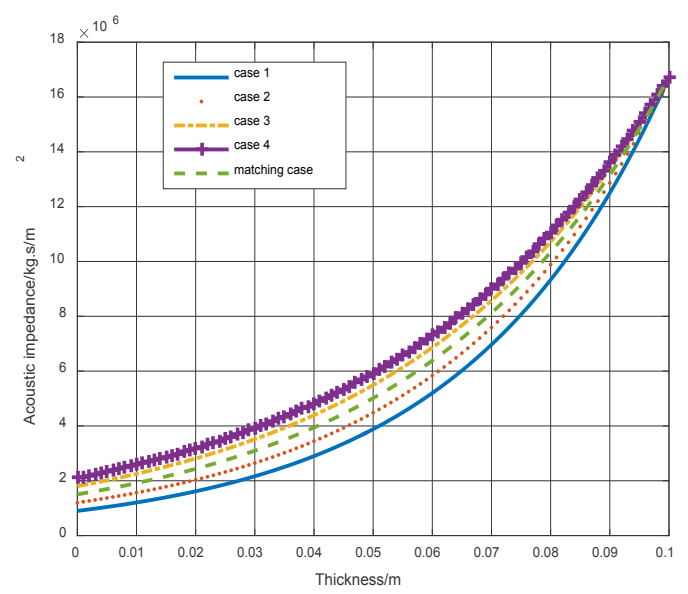

(a) Acoustic impedance distributions of the medium.

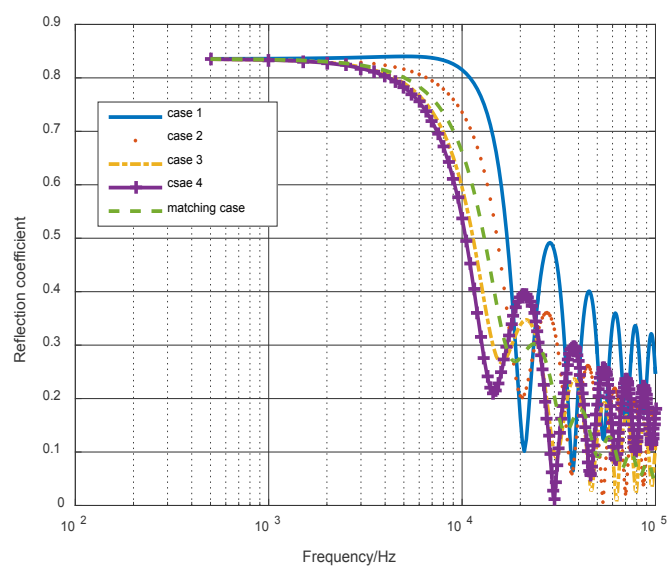

(b) Reflection coefficients of different cases.

Fig. 2. The simulation result of acoustic impedance mismatch between boundary of medium and water.

Table 1. Acoustic impedance mismatch parameter.

\begin{tabular}{|c|c|c|c|c|}
\hline Case & $\mathbf{a}=\mathbf{1 0} \%$ & $\mathbf{a}=\mathbf{2 0} \%$ & $\mathbf{a}=\mathbf{3 0} \%$ & $\mathbf{a}=\mathbf{4 0} \%$ \\
\hline $\mathrm{R}$ & 0.2165 & 0.2246 & 0.2408 & 0.2599 \\
\hline$\Delta$ & $1.86 \%$ & $1.81 \%$ & $9.16 \%$ & $17.82 \%$ \\
\hline
\end{tabular}

where $R$ is the average value of the reflection coefficient obtained under the condition of each mismatch case, and $R_{m}$ means the average value of the reflection coefficient obtained from matching case. In Table 1, as the degree of acoustic impedance mismatch increases, the value of $\Delta$ becomes larger. When acoustic impedance mismatch between boundary of medium and water is $20 \%$, the reflection coefficient increases $1.81 \%$. When acoustic impedance mismatch between boundary of medium and water is $30 \%, \Delta$ increases more than $9 \%$. When acoustic impedance mismatch between boundary of medium and water, the degree of acoustic impedance mismatch should be less than $20 \%$, which reduces the difficulty of preparing the medium and makes the deviation of acoustic characteristic less than $2 \%$. 


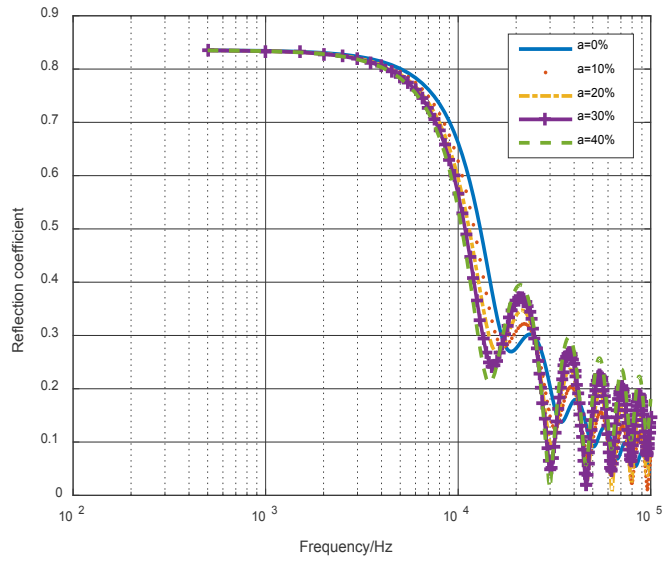

Fig. 3. Reflection coefficients of different cases.

\subsection{Acoustic impedance mismatch between boundary of medium and aluminum}

In this section, the acoustic characteristics of acoustic impedance mismatch between boundary of medium and aluminum are attained by comparing the numerical results of different mismatch cases.

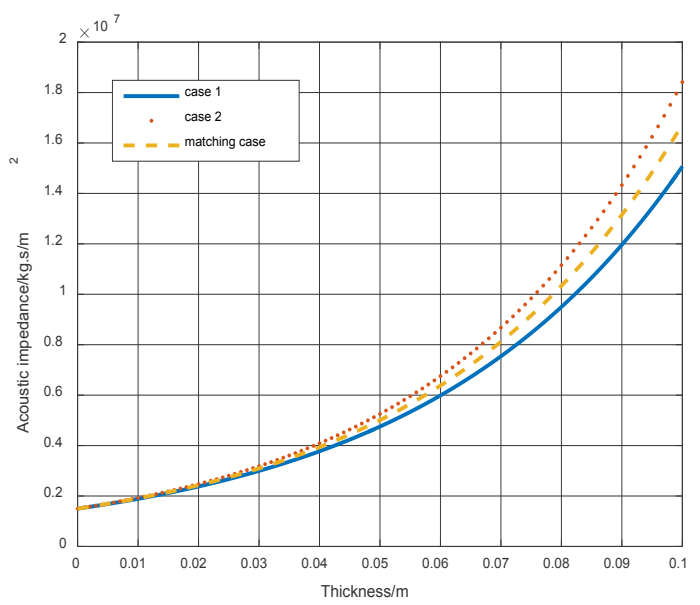

(a) Acoustic impedance distributions of the medium.

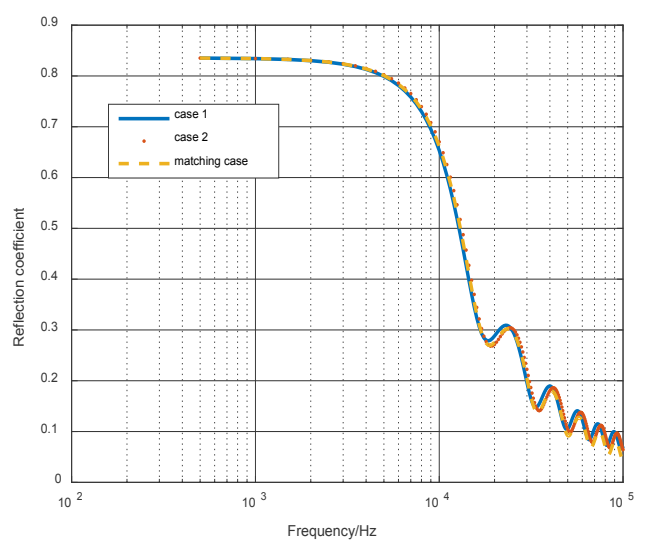

(b) Reflection coefficients of different cases.

Fig. 4. The simulation result of acoustic impedance mismatch between boundary of medium and aluminum.

$Z_{n}$ represents the acoustic impedance at the interface between the medium with gradient change of acoustic impedance and aluminum, $Z_{a}$ means the acoustic impedance of aluminum. Two mismatch cases are as follows: case $1: Z_{n} / Z_{a}=0.9$, case $2: Z_{n} / Z_{a}=1.1$, and a matching case: $Z_{\mathrm{n}} / Z_{\mathrm{a}}=1.0$. The acoustic impedance at the boundary of the medium and water is the same as the acoustic impedance of water. Acoustic impedance distributions of the medium are shown in Fig.4(a). The simulation results of three cases within the frequency range of $0-100 \mathrm{kHz}$ are shown in Fig.4(b).

In Fig.4(b), three cases are almost the same in the entire frequency. Within the frequency range of $0-10$ $\mathrm{kHz}$, they remain around 0.835 . Within the frequency range of $10 \mathrm{kHz}-100 \mathrm{kHz}$, all cases oscillate down. After calculation, $\bar{R}_{1}=0.2287$, and $\bar{R}_{2}=0.2313$. The data obtained from the matching case is 0.2206 . When acoustic impedance mismatch between boundary of medium and aluminum, it has little effect on acoustic characteristics. Because the medium which the wave first contacts in the process of sound wave propagation has the greatest influence on the propagation.

\subsection{Acoustic impedance mismatch between boundary of medium and both sides}

In this section, the acoustic characteristics of acoustic impedance mismatch between boundary of medium and aluminum are attained by comparing the numerical results of different mismatch cases.

Similarly, four mismatch cases are as follows: case 1: $\mathrm{Z}_{0} / \mathrm{Z}_{\mathrm{w}}=0.8, \mathrm{Z}_{\mathrm{n}} / \mathrm{Z}_{\mathrm{a}}=0.9$, case $2: \mathrm{Z}_{0} / \mathrm{Z}_{\mathrm{w}}=0.8, \mathrm{Z}_{\mathrm{n}} / \mathrm{Z}_{\mathrm{a}}=1.1$, case 3: $Z_{0} / Z_{w}=1.2, Z_{n} / Z_{a}=0.9$, case $4: Z_{0} / Z_{w}=1.2, Z_{n} / Z_{a}=1.1$, and a matching case: $Z_{0} / Z_{\mathrm{w}}=1.0, Z_{\mathrm{n}} / Z_{\mathrm{a}}=1.0$. Acoustic impedance distributions of the medium are shown in Fig.5(a). The simulation results of five cases within the frequency range of $0-100 \mathrm{kHz}$ are shown in Fig.5(b).In Fig.5(b), three cases are basically the same over the entire frequency. Within the frequency range of $0-10$ $\mathrm{kHz}$, three cases remain around 0.84 . Within the frequency range of $10 \mathrm{kHz}-100 \mathrm{kHz}$, they all have varying degrees of oscillations.

After calculation, $\bar{R}_{1}=0.2697, \bar{R}_{2}=0.2729$, $\bar{R}_{3}=0.2301$ and $\bar{R}_{4}=0.2317$. The data of the matching case is 0.2206 . It is observed that $\bar{R}_{3}$ and $\bar{R}_{4}$ are close to the matching case. Taking into account that case 3 will reduce the difficulty of preparing the medium. Above all, it is worth to explore case 3 . In order to calculate the effects of different acoustic impedance mismatch on the acoustic characteristics of the medium, we define the same as in the section 3.1 and $b=\left(Z_{a}-Z_{n}\right) / Z_{a}$, which mean the degree of acoustic impedance mismatch between the medium and both sides. The reflection coefficients are simulated within the frequency range of $0-100 \mathrm{kHz}$, and the results are shown in Fig.6.

In Fig.6, five cases are almost unchanged within the frequency range of $0-5 \mathrm{kHz}$. As the frequency increases, all cases are accompanied by different degrees of oscillations. After calculation, the average values of the reflection coefficients of four mismatch cases are 0.2247 , $0.253,0.2954$, and 0.3484 . The data obtained from the matching case is 0.2206 . The numerical values of each case are shown in Table 2. 


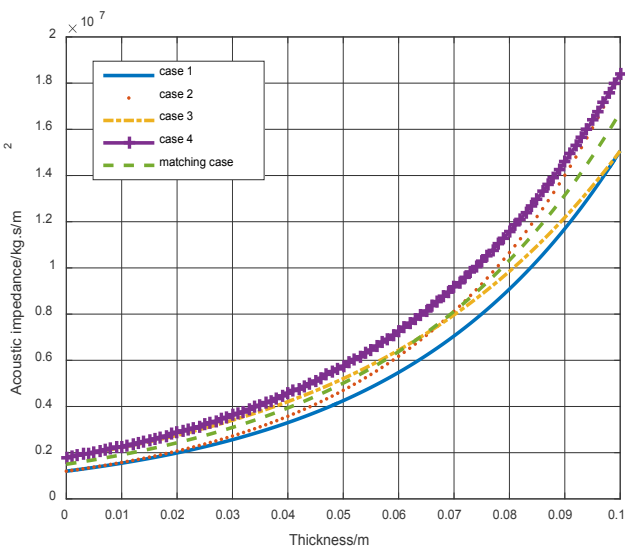

(a) Acoustic impedance distributions of the medium.

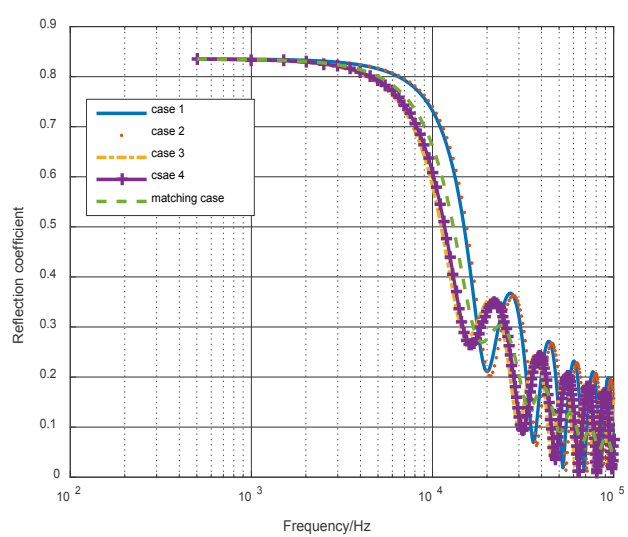

(b) Reflection coefficients of different cases.

Fig. 5. The simulation result of acoustic impedance mismatch between boundary of medium and both sides.

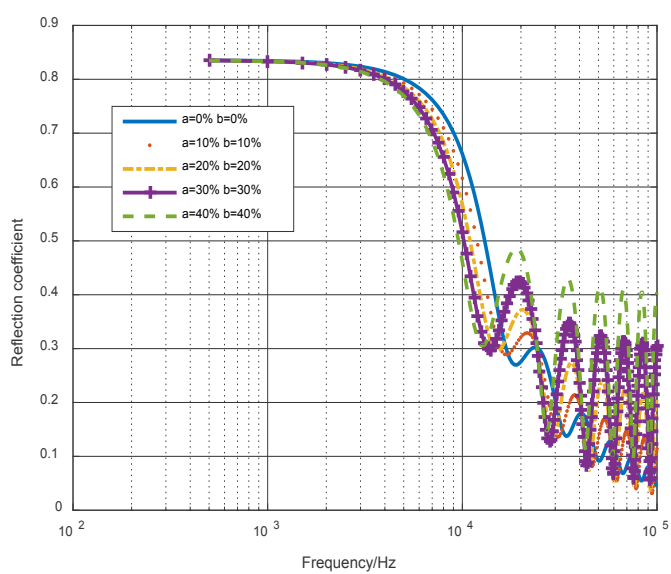

Fig. 6. Reflection coefficients of different cases.

Table 2. Acoustic impedance mismatch parameter.

\begin{tabular}{|c|c|c|c|c|}
\hline Case & $\begin{array}{c}\mathbf{a}=\mathbf{1 0 \%} \\
\mathbf{b}=\mathbf{1 0 \%}\end{array}$ & $\begin{array}{c}\mathbf{a}=\mathbf{2 0 \%} \\
\mathbf{b}=\mathbf{2 0} \%\end{array}$ & $\begin{array}{c}\mathbf{a}=\mathbf{3 0 \%} \\
\mathbf{b}=\mathbf{3 0 \%}\end{array}$ & $\begin{array}{c}\mathbf{a}=\mathbf{4 0 \%} \\
\mathbf{b}=\mathbf{4 0 \%}\end{array}$ \\
\hline$R$ & 0.2247 & 0.253 & 0.2954 & 0.3484 \\
\hline$\Delta$ & $1.86 \%$ & $14.67 \%$ & $33.88 \%$ & $57.94 \%$ \\
\hline
\end{tabular}

In Table 2, as the degree of acoustic impedance mismatch increases, the value of $\Delta$ becomes larger. When acoustic impedance mismatch between boundary of medium and both sides is $10 \%$, the reflection coefficient increases $1.86 \%$. When acoustic impedance mismatch between boundary of medium and both sides is $20 \%, \Delta$ increases more than $10 \%$. When acoustic impedance mismatch between boundary of medium and both sides, the degree of acoustic impedance mismatch should be less than $10 \%$, which reduces the difficulty of preparing the medium and makes the deviation of acoustic characteristic less than $2 \%$.

\section{Conclusions}

The work presented in this paper establishes the acoustical wave propagation model in the medium with gradient change of acoustic impedance. The medium with gradient change of acoustic impedance is difficult to match the acoustic impedance on both sides of the medium. By comparing the numerical result of different acoustic impedance mismatch cases, the influence of acoustic impedance mismatch on the acoustic characteristics was obtained. Three acoustic impedance mismatch cases are computed and compared in this paper. When acoustic impedance mismatch between boundary of $m$ edium and water, the degree of acoustic impedance mismatch should be less than $20 \%$, which makes the deviation of acoustic characteristic less than $2 \%$. When acoustic impedance mismatch between boundary of medium and aluminum, it has little effect on the acoustic characteristics. When acoustic impedance mismatch between boundary of medium and both sides, the degree of acoustic impedance mismatch should be less than $10 \%$, which makes the deviation of acoustic characteristic less than $2 \%$.

\section{References}

1. D. Takahashi, J. Acoust. Soc. Jap. 54, 40-46 (1997)

2. Z. Gerhard, A. Schievenbusch, Mateials Science Forum. 308-311, 533-538 (1999)

3. P. C. Pedersen, O. Tretiak, P. He, J. Acoust. Soc. Am. 72, 327-336, (1982)

4. G. Caviglia, A. Morro, Int. J. Eng. Sci. 38, 847-863, (2000)

5. D. Li, L. Zigoneanu, B. I. Popa, J. Acoust. Soc. Am. 132, 2823, (2012)

6. G. Feng, W. Liu, Sensors, 13, 13543-13559, (2013)

7. K. Kimura, K. Yoneya, H. Ohigashi, Jap. Phys. J. Appl. Phys. 27, 547-551(2014)

8. A.A.E.Ouahabi, V.V.Krylov, D.J.O'Boy, European Congress and Exposition on Noise Control Engineeing, 989-994(2015)

9. S. Setunge, N. Gamage, Springer Singapore, (2016)

10. D. Xu, X. Cheng, S. Huang, Compos. Sci. Technol. 122, 90-96, (2016) 